\title{
Eficácia acaricida de uma emulsão contendo $10 \%$ de óleo de nim (Azadirachta indica) no controle de Psoroptes ovis em coelhos naturalmente infestados ${ }^{1}$
}

\begin{abstract}
Julio I. Fernandes ${ }^{2 *}$, Guilherme G. Verocai ${ }^{3}$, Francisco A. Ribeiro ${ }^{4}$, Raquel M.P.S. Melo $^{5}$, Thais R. Correia ${ }^{6}$, Cristiano C.P. Veiga ${ }^{7}$, Vanessa P.C. Vieira ${ }^{8}$ e Fabio B. Scott ${ }^{9}$

ABSTRACT.- Fernandes J.I., Verocai G.G., Ribeiro F.A., Melo R.M.P.S., Correia T.R.,Veiga C.C.P., Vieira V.P.C. \& Scott F.B. 2012. [Miticidal efficacy of an emulsion containing $10 \%$ neem (Azadirachtaindica) oil for control of Psoroptes ovis in naturally infested rabbits.] Eficácia acaricida de uma emulsão contendo $10 \%$ de óleo de Nim (Azadirachta indica) no controle de Psoroptes ovis em coelhos naturalmente infestados. Pesquisa Veterinária Brasileira 32(12):1253-1256. Faculdade de Medicina Veterinária, Universidade Federal do Pará, BR-316 Km 61, Castanhal, PA 68740-970.Brazil. E-mail: vetjulio@yahoo.com.br

The objective of the present study was to evaluate the efficacy of an emulsion containing $10 \%$ of neem (Azadirachta indica) oil on the control of Psoroptes ovis in naturally infested rabbits. Twelve rabbits were randomly divided in two groups of six animals. The control group remained without treatment; while animals in the other group were treated with the $10 \%$ neem extract formulation by spraying both ears daily for seven consecutive days. The animals were evaluated daily for the presence of adverse effects. Material from ears all animals was collected on days $+3,+7,+14,+21,+28$ and +35 , and evaluated for the presence of living mites. Animals from control group presented mites in both ears along all days of observation. The treated group presented an efficacy of $41.7 \%$ on day +3 and $100 \%$ from day +7 to +35 . The product containing $10 \%$ neem oil has demonstrated to be effective for the treatment of psoroptic mange on rabbits. However, treated animals presented dermatological reaction such as alopecia and hyperemia at the site of application, varying from low to medium severity.
\end{abstract}

INDEX TERMS: Azadirachta indica, control, Oryctolagus cuniculus, phytotherapy, psoroptic mange.

RESUMO.- 0 objetivo do presente estudo foi avaliar a eficácia de uma emulsão contendo $10 \%$ de óleo de nim, Azadirachta indica, no controle de Psoroptes ovis em coelhos naturalmente infestados. Foram utilizados 12 coelhos separados aleatoriamente em dois grupos de seis animais cada. 0 grupo controle permaneceu sem tratamento, enquanto que o grupo tratado recebeu a formulação em teste, contendo

\footnotetext{
${ }^{1}$ Recebido em 25 de abril de 2012.

Aceito para a publicação em 11 de setembro de 2012.

${ }^{2}$ Faculdade de Medicina Veterinária, Universidade Federal do Pará (UFPA), BR-316 Km 61, Castanhal, PA 68740-970, Brasil. *Autor para correspondência: vetjulio@yahoo.com.br

${ }^{3}$ Department of Ecosystem and Public Health, Faculty of Veterinary Medicine, University of Calgary, 3330 Hospital Drive, NW, Calgary, AB, T2N 4N1 Canada. E-mail: gui.verocai@ucalgary.ca

${ }^{4}$ Clínica Veterinária Vaz Lobo, Av. Vicente de Carvalho 316, Vicente de Carvalho, Rio de Janeiro, RJ 21371-148, Brasil E-mail: fran.ribeirovet@ gmail.com

${ }^{5}$ Curso de Zootecnia, Universidade Federal de Alagoas, Av. Manoel Seve-
}

$10 \%$ de Nim, borrifando ambos os condutos auditivos, uma vez ao dia, por sete dias consecutivos. Os animais foram avaliados diariamente para observação de possíveis efeitos adversos do produto. Nos dias $+3,+7,+14,+21,+28$ e +35 foi coletado material de todos os animais para avaliação da presença de ácaros vivos. Os coelhos do grupo controle apresentavam ácaros em ambos os condutos auditivos em

\footnotetext{
rino Barbosa s/n, Bom Sucesso, Arapiraca, AL 57309-005, Brasil. E-mail: raquelmpsm@gmail.com

${ }^{6}$ Faculdade de Medicina Veterinária de Valença, Fundação Educacional Dom André Arcoverde, Centro de Ensino Superior de Valença, Rua Sargento Vitor Hugo 161, Valença, RJ 27600-000, Brasil. E-mail: thaisrca@gmail.com

${ }^{7}$ Hospital Veterinário, Universidade Federal Rural do Rio de Janeiro (UFRRJ), BR 465 Km 7, Seropédica, RJ 23890-000, Brasil, e Doutorando do PPGCRA/UFF. E-mail: radiovet@ufrrj.br

${ }^{8}$ Escola de Medicina Veterinária e Zootecnia, Universidade Federal do Tocantins (UFT), BR $153 \mathrm{Km} \mathrm{112,} \mathrm{Araguaína,} \mathrm{T0} \mathrm{77800-970,} \mathrm{Brasil.}$ E-mail: vanessarural@hotmail.com

${ }^{9}$ Instituto de Veterinária, (UFRRJ), Seropédica, RJ. E-mail: scott@ufrrj.br
} 
todos os dias de observação. 0 grupo tratado apresentou eficácia de $41,7 \%$ no dia +3 e $100 \%$ a partir do dia +7 até o dia +35 . 0 produto demonstrou ser eficaz no tratamento da sarna psoróptica em coelhos. Entretanto, todos os animais tratados apresentaram reações dermatológicas, tais como alopecia e hiperemia no local de aplicação do produto, variando de baixa a média severidade.

TERMOS DE INDEXAÇÃO: Azadirachta indica,controle, fitoterapia, Oryctolagus cuniculus, sarna psoróptica.

\section{INTRODUÇÃO}

O coelho doméstico (Oryctolagus cuniculus) é considerado um animal economicamente importante por ser utilizado pela indústria na produção de carne, pele e couro. 0 coelho também fornece adubo orgânico, que possui um alto valor no mercado, além do cérebro, que pode ser desidratado e moído para obtenção de tromboplastina, um agente coagulador do sangue, além de serem considerados animais de companhia.

Distúrbios dermatológicos são relativamente comuns nesta espécie, interferindo na qualidade de vida dos mesmos (White et al. 2002). Dentre as diversas etiologias que levam as lesões dermatológicas, os parasitos são os que prevalecem sobre bactérias, vírus e fungos.

O ácaro Psoroptes ovis é ectoparasito obrigatório de equídeos, bovinos, caprinos, ovinos e coelhos, assim como de diversas espécies de mamíferos selvagens (Wall \& Kolbe 2006).

Nos coelhos, $P$. ovis é o ectoparasito mais relatado, encontrado principalmente nos condutos auditivos, levando a quadros de otite clínica onde são observadas crostas, ulcerações e hiperemia com formação de tecido de granulação (Chen et al. 2000).

Menos freqüentemente, a infestação pode se mostrar generalizada, acometendo todo o corpo do hospedeiro (Cutler 1998, Bates 1999), gerando dermatites debilitantes, alopecia, formações de crostas e intenso prurido (White et al. 2002, Siegfried et al. 2004).Em casos extremos o parasitismo pode resultar em alterações neurológicas (White et al. 2002).

O diagnóstico é confirmado examinando-se as crostas oriundas das lesões ao microscópio estereoscópio ou microscópio óptico, onde podem ser visualizados diversos estágios do ácaro(White et al. 2002).

Diversos protocolos terapêuticos têm sido propostos para o tratamento desta parasitose em coelhos, podendo ser utilizado tratamento tópico ou sistêmico, não sendo necessária a remoção mecânica das crostas (White et al. 2002). Dentre as principais drogas utilizadas no controle do ácaro em coelhos, destacam-se a ivermectina, moxidectina (Morrisey 1996, White et al. 2002), eprinomectina (Pan et al. 2006) epermetrina (Melo et al. 2008). Tais drogas são muitas vezes empregadas em coelhos, mesmo não contendo indicação na bula, o que acarreta riscos no que se refere à ausência de informações sobre o período residual na carne, não informando sobre o período de carência a ser observado.

Acaricidas têm sido amplamente empregados ao longo dos anos de maneira eficaz e econômica. Entretanto, seu uso indiscriminado tem levado ao aparecimento de populações resistentes, de contaminação ambiental e quadros de intoxicação nos homens e nos animais fazendo com que novas modalidades de controle sejam desenvolvidas (Wall 2007). Dentro destas modalidades de controle merece destaque a utilização de biopesticidas.

Um país como o Brasil, com tantas peculiaridades e pluralidade climáticas e geográficas, abriga uma diversidade enorme de plantas que podem ser utilizadas no controle de artrópodes. Pertencente a família Meliaceae, a planta de origem indiana Azadirachta indica, popularmente conhecida como nim, que vem sendo empregada no controle de diversas pragas agrícolas com sucesso. A planta $A$. indica possui diversas atividades terapêuticas destacando-se sua alta eficácia e segurança, pois apresenta baixa toxidade para os mamíferos (Isman 1997, Menezes 2005), embora Ahmed \& Grainge (1986) tenham relatado toxidade em coelhos quando o extrato contendo nim foi administrado por via oral.

Diversos processos podem ser empregados para a extração de diferentes compostos com atividades biológicas. No entanto, apenas alguns desses compostos apresentam eficácia como pesticidas. Dentre estes, destacam-se a azadiractina, salanima e nimbina (Santos \& Andrade 2000, Abdel-Shafy \& Zayed 2002, Menezes 2005), atuando principalmente como inibidores de desenvolvimento de artrópodes ou impedindo a alimentação dos mesmos (Mordue \& Blackwell 1993, Martinez 2002). O objetivo do presente estudo foi avaliar a eficácia de uma emulsão aquosa contendo $10 \%$ de óleo de nim, $A$. indica, no controle de $P$. ovis em coelhos naturalmente infestados.

\section{MATERIAL E MÉTODOS}

Foram utilizados 12 coelhos, machos, adultos, pertencentes ao Laboratório de Quimioterapia Experimental em Parasitologia Veterinária (LQEPV), do Departamento de Parasitologia Animal, do Instituto de Veterinária, da Universidade Federal Rural do Rio de Janeiro, Município de Seropédica, Rio de Janeiro. Os animais foram adquiridos no setor de cunicultura do Instituto de Zootecnia da mesma Universidade para serem utilizados na manutenção de colônia de carrapatos da espécie Rhipicephalus sanguineus do LQEPV.

Todos os coelhos estavam naturalmente infestados por Psoroptes ovis (Hering, 1838), diagnosticados a partir da visualização dos ácaros com auxílio de microscópio estereoscópico, a partir de raspados cutâneos efetuados nas orelhas. Os animais foram divididos aleatoriamente em dois grupos com seis animais cada. Um grupo permaneceu sem tratamento, grupo controle, e o outro foi medicado. A dosagem, frequência e duração do tratamento foram estipuladas de acordo com trabalhos que utilizaram o extrato de nim, no controle de outros parasitos e em diferentes espécies, uma vez que até o presente momento não há relatos na literatura sobre a utilização do medicamento em coelhos infestados por $P$. ovis (Abdel-Ghaffar et al. 2008, Fernandes et al. 2010, Schmahl et al. 2010).

Nenhum produto parasiticida foi empregado nos animais ou no ambiente por até 60 dias antes do tratamento. Os animais de cada grupo foram mantidos em gaiolas suspensas dentro de ambientes separados localizadas no próprio laboratório com água e comida ad libitum durante todo o período de experimentação. 
Foi utilizada a emulsão de nim, a partir do óleo de nim puro ${ }^{10}$. Para o preparo da emulsão foi acrescentado a uma parte do óleo, tween 80 (emulsificante) na concentração de 30\%. Posteriormente, a solução foi levada a um agitador magnético com aquecimento, onde permanecia por 10 minutos a uma temperatura de $40^{\circ} \mathrm{C}$, completando até a quantidade desejada com água.

Nos animais pertencentes ao grupo medicado foram aspergidos dois mililitros em cada conduto auditivo com a emulsão aquosa experimental contendo $10 \%$ de óleo de nim. Foram realizadas aplicações diárias, durante os sete primeiros dias. A dosagem e a frequência de aplicação do produto utilizado no presente estudo foram estipuladas seguindo a utilização de produtos químicos comerciais, clinicamente testados, empregados no controle de parasitos auriculares em animais de companhia, uma vez que não existe ainda um protocolo para a utilização de biopesticidas em coelhos.

Foram realizadas avaliações clínicas diárias nos animais para registro de eventuais reações adversas causadas pelo produto e, também para avaliara regressão das lesões auriculares provocadas pela ação dos ácaros. Todos os animais foram reexaminados nos dias $+3,+7,+14,+21,+28$ e +35 após o primeiro dia do tratamento. 0 material foi avaliado com auxílio de microscópio estereoscópico. Não foi realizada limpeza ou retirada das crostas, antes ou durante o período do tratamento. A eficácia do tratamento do produto foi calculada em cada orelha através da seguinte fórmula: (número de orelhas infestadas com os ácaros vivos antes do tratamento - número de orelhas infestadas com os ácaros vivos após o tratamento) / (número de orelhas infestadas com os ácaros vivos antes do tratamento) X100 (Abbott 1987). A análise estatística dos resultados foi realizada através do Teste do Qui-quadrado $\left(\chi^{2}\right)$ corrigido (Correção de Yates) (Sampaio 1998).

\section{RESULTADOS E DISCUSSÃO}

Os coelhos pertencentes ao grupo controle apresentaram-se infestados por P. ovis, em ambos os condutos auditivos, durante todo período experimental. Já nos animais medicados, os resultados encontram-se listados no Quadro 1. No grupo tratado, até o dia +3 , todos os animais permaneciam parasitados. Já a partir do dia +7 , não foram mais encontrados parasitos vivos nos animais medicados.

Como cada orelha foi considerada uma unidade experimental, observou-se no dia +3 , um total de sete orelhas positivas, correspondendo a uma eficácia de $41,66 \%$. Já nos dias subsequentes de observação, não foram encontrados parasitos vivos, em nenhuma das orelhas dos animais medicados indicando uma eficácia de $100 \%$. Através do teste de $\chi^{2}$ corrigido (Yates) obteve-se o valor 20,17, com $(p<0,05)$. Clinicamente notou-se regressão das lesões auriculares a partir do dia +7 .

Embora seja relatada por muitos pesquisadores como uma planta muito segura para mamíferos, todos os animais apresentaram reações adversas a formulação, provavelmente pelo uso contínuo da emulsão aquosa. As lesões eram caracterizadas por leve a moderado prurido, alopecia e hiperemia nos locais onde foi aplicado o produto. As lesões, entretanto, regrediram após o término do tratamento. Já a pele dos animais voltou à normalidade20 dias após a suspensão do medicamento.

Atualmente, diversos protocolos terapêuticos têm sido empregados no controle da sarna psoróptica em coelhos,

\footnotetext{
${ }^{10}$ Laboratório Natural Rural ${ }^{\circledR}$.
}

\section{Quadro 1. Presença de Psoroptes ovis vivos nas orelhas direita e esquerda dos coelhos pertencentes ao grupo controle e ao tratado com a formulação em teste contendo $10 \%$ de extrato aquoso de Nim, Azadirachta indica, antes e após o tratamento}

\begin{tabular}{|c|c|c|c|c|c|c|c|c|c|c|c|c|c|c|}
\hline \multirow{3}{*}{$\begin{array}{l}\text { Grupos/ } \\
\text { Animais }\end{array}$} & \multicolumn{14}{|c|}{ Presença de ácaros vivos por dia de desafio } \\
\hline & \multicolumn{2}{|c|}{ Dia 0} & \multicolumn{2}{|c|}{ Dia+3 } & \multicolumn{2}{|c|}{ Dia+7 } & \multicolumn{2}{|c|}{ Dia+14 } & \multicolumn{2}{|c|}{ Dia+21 } & \multicolumn{2}{|c|}{$\mathrm{Dia}+28$} & \multicolumn{2}{|c|}{$\mathrm{Dia}+35$} \\
\hline & $\mathrm{OD}^{\mathrm{a}}$ & $\mathrm{OE}^{\mathrm{b}}$ & $\mathrm{OD}$ & $\overline{\mathrm{OE}}$ & $\mathrm{OD}$ & $\mathrm{OE}$ & $\mathrm{OD}$ & $\mathrm{OE}$ & $\mathrm{OD}$ & $\mathrm{OE}$ & $\mathrm{OD}$ & $\mathrm{OE}$ & $\mathrm{OD}$ & $\mathrm{OE}$ \\
\hline \multicolumn{15}{|l|}{ Tratado } \\
\hline 01 & $t^{3}$ & + & + & -4 & - & - & - & - & - & - & - & - & - & - \\
\hline 02 & + & + & - & + & - & - & - & - & - & - & - & - & - & - \\
\hline 03 & + & + & + & - & - & - & - & - & - & - & - & - & - & - \\
\hline 04 & + & + & - & + & - & - & - & - & - & - & - & - & - & - \\
\hline 05 & + & + & + & + & - & - & - & - & - & - & - & - & - & - \\
\hline 06 & + & + & + & - & - & - & - & - & - & - & - & - & - & - \\
\hline \multicolumn{15}{|l|}{ Controle } \\
\hline 07 & & & & & & & & & & & & & & \\
\hline 08 & + & + & + & + & + & + & + & + & + & + & + & + & + & + \\
\hline 09 & + & + & + & + & + & + & + & + & + & + & + & + & + & + \\
\hline 10 & + & + & + & + & + & + & + & + & + & + & + & + & + & + \\
\hline 11 & + & + & + & + & + & + & + & + & + & + & + & + & + & + \\
\hline 12 & + & + & + & + & + & + & + & + & + & + & + & + & + & + \\
\hline
\end{tabular}

$\overline{\text { a }}$ Orelha direita, ${ }^{\mathrm{b}}$ Orelha esquerda.

destacando-se o tratamento sistêmico com ivermectina (Ferrero et al. 1994) ou moxidectina (Wagner \& Wendlberger 2000), ou tratamentos tópicos, utilizando piretróides (Melo et al. 2008) ou organofosforados, que embora apresentem altos níveis de eficácia, podem acarretar efeitos tóxicos nos animais, além da questão do resíduo na carne (O’Brien 1999, Hansen et al. 2005, Nero et al. 2007).

Outros autores relatam o emprego do óleo de nim em coelhos naturalmente infestados com $P$. ovis com resultados distintos. Os resultados do presente estudo são similares aos descritos por Dakshinkar et al.(1992), quando utilizaram extratos de A. indica, Annona squamosa (fruta-do-conde) e Allium sativum (alho), encontrando resultados positivos a partir do dia +4 , não sendo mais observado ácaros adultos, ninfas ou ovos, sendo confirmados através de avaliações realizadas nos dias +10 e +20 .

Maske \& Kolte (1999) compararam o uso da ivermectina com uma formulação contendo os extratos de $A$. indica, Cedrus deodara (Cedro do Himalaia) e Embelia ribes (Vidanga ou falsa pimenta-negra) para o controle de P. ovis em coelhos naturalmente infestados. Foi observada melhor eficácia da associação dos fitoterápicos em relação ao emprego da ivermectina. Os resultados são similares ao estudo, embora tenha sido usada apenas a emulsão contendo $10 \%$ de óleo de nim. Assim, a utilização da planta A. indica associada ou não a outros fitoterápicos mostrou-se eficaz no controle dos ácaros.

Ao empregarem em coelhos infestados naturalmente por P. ovis uma pomada contendo óleo de nim a 5\%, Joshi et al. (2000) relatam que tal formulação não foi eficaz no controle dos ácaros. Tais resultados podem ser pela menor concentração empregada ou por sua apresentação, não permitindo que o produto tivesse boa dispersão, sendo estes resultados inferiores aos do presente estudo.

0 uso de plantas com propriedades medicinais vêm aumentando no Brasil devido à ampla divulgação de técnicas de cultura orgânica e seus benefícios que se traduz por 
alimentos seguros, mais saudáveis e isentos de defensivos químicos. Porém, antes de empregar compostos biopesticidas, a eficácia e segurança quanto sua utilização deve ser testada.

\section{CONCLUSÃO}

Os resultados do presente trabalho permitem concluir que a formulação aquosa contendo $10 \%$ de óleo de nim possui eficácia contra o ácaro Psoroptes ovis em coelhos. Entretanto, novos estudos devem ser realizados com o objetivo de se evitar os efeitos colaterais desencadeados após o emprego da formulação na concentração $10 \%$ de aplicação diária, seja diminuindo a concentração utilizada, aumentando o intervalo entre as aplicações ou diminuindo o número de aplicações.

\section{REFERÊNCIAS}

Abdel-Ghaffar F., Al-Quraishy S., Sobhy H. \& Semmler M. 2008. Neem seed extract shampoo, Wash Away Louse ${ }^{\circledR}$, an effective plant agent against Sarcoptes scabiei mites infesting dogs in Egypt. Parasitol. Res. 104:145-148.

Abdel-Shafy S. \& Zayed A.A. 2002. In vitro acaricidal effect of plant extract of Neem seed oil (Azadirachtaindica) on egg, immature, and adult stages of Hyalomma anatolicum excavatum (Ixodoidea: Ixodidae). Vet. Parasitol. 106(1): 89-96.

Abbott W.S. 1987. A method of computing the effectiveness of an insecticide. J. Am. Mosq. Control Assoc. 3(2):302-303.

Ahmed S. \& Grainge M. 1986. Potential of the Neem Tree (Azadirachta indica) for pest control and rural development. Econ. Bot. 40(2):201-209.

Bates P.G. 1999. Inter and intra-specific variation within the genus Psoroptes (Acari: Psoroptidae). Vet. Parasitol. 83(3/4):201-217.

Chen L.P., Chung T.K. \& Lin S.C. 2000. Psoroptes cuniculi infestation in rabbits in central Taiwan. J. Chin. Soc. Vet. Sci. 26(4):284-292.

Cutler S.L. 1998. Ectopic Psoroptes cuniculi infestation in a pet rabbit. J. Small Anim. Pract. 39(2):86-87.

Dakshinkar N.P., Sharma S.R., Kothekar M.D., Sapre V.A. \& Gore A.K. 1992. Effect of crude extracts of three indigenous plants against ear mange of rabbits. Indian Vet. Med. J. 16(4):288-291.

Fernandes J.I., Correia T.R., Ribeiro F.A., Cid Y.P., Tavares P.V. \& Scott F.B. 2010. Eficácia do nim (Azadirachta indica) no controle de Otodectes cynotis (Hering, 1838) em cães. Revta Bras. Med. Vet. 32:55-58.

Ferrero O., Rebuelto M., Albarellos G. \& Hallu R. 1994. Efficacy of ivermectin in the treatment of rabbit ear canker. Vet. Argentina 11(104):242244.

Guerrini V.H. \& Kriticos C.M. 1998. Effects of Azadirachtin on Ctenocephalides felis in the dog and the cat. Vet. Parasitol.74(2/4):289-297.

Hansen O., Gall Y., Pfister K. \& Beck W. 2005. Efficacy of a formulation containimg imidacloprid and moxidectin against naturally acquired ear mite infestations (Psoroptes cuniculi) in rabbits. J. Appl. Res. Vet. Med. 3(4):281-286.
Isman M.B. 1997. Neem insecticides. Pesticide Outlook 8(5):32-38.

Joshi S.S., Dakshinkar N.P., Sapre V.A. \& Sarode D.B. 2000. Evaluation of herbal medicaments in psoroptic mange of rabbits. Indian Vet. J. 77(8): 706-708.

Martinez S.S. 2002. 0 nim, Azadirachta indica: natureza, usos múltiplos, produção. Inst. Agron. Paraná, Londrina, p.142.

Maske D.K. \& Kolte S.W. 1999. Studies on life-cycle of psoroptic mange mite in rabbits and chemotherapy under experimental conditions. J. Vet. Parasitol. 13(1):45-47.

Melo R.M.P.S., Fernandes J.I., Cruz V.P., Ribeiro F.A., Botelho M.C.S.N., Verocai G.G. \& Scott F.B. 2008. Eficácia do piretróide permetrina no controle de Psoroptes ovis (Hering, 1838) (Acari: Psoroptidae) em coelhos (Oryctolagus cuniculus) naturalmente infestados. Revta Bras. Parasitol. Vet. 17(1):55-58.

Menezes E.L.A. 2005. Inseticidas botânicos: seus princípios ativos, modo de ação e uso agrícola. Embrapa Agrobiologia, Seropédica, RJ. 58p.

Mordue A.J. \& Blackwell A. 1993. Azadirachtin: An update. J. Insect Physiol. 39(11):903-924.

Morrisey J.K. 1996. Parasites of ferrets, rabbits, and rodents. Sem. Avian and Exotic Pet Med.5(2):106-114.

Nero L.A., Mattos M.R., Beloti V., Barros M.A.F., Pontes Netto D. \& Franco B.D.G.M. 2007. Organofosforados e carbamatos no leite produzido em quatro regiões leiteiras no Brasil: ocorrência e ação sobre Listeria monocytogenes e Salmonella spp. Ciênc. Tecnol. Aliment. 27(1):201-204.

O’Brien D.J. 1999. Treatment of psoroptic mange with reference to epidemiology and history. Vet. Parasitol.83(3/4):177-185.

Pan B., Wang M., Xu F., Wang Y., Dong Y. \& Pan Z. 2006. Efficacy of an injectable formulation of eprinomectin against Psoroptes cuniculi, the ear mange mite in rabbits. Vet. Parasitol. 137(3/4):386-390.

Sampaio I.B. M. 1998. Estatística Aplicada à Experimentação Animal. Fundação de Ensino e Pesquisa de Medicina Veterinária e Zootecnia, Belo Horizonte. 265p.

Santos L.U. \& Andrade C.F.S. 2000. Azadirachta indica: a árvore do nim e o controle de piolhos. Departamento de Zoologia, Instituto de Biologia, Unicamp, Campinas.

Schmahl G., Al-Rasheid K.A.S., Abdel-Ghaffar F., Klimpel S. \& Mehlhorn H. 2010. The efficacy of neem seed extracts (Tre-san ${ }^{\circledR}$, MiteStop $^{\circledR}$ ) on a broad spectrum of pests and parasites. Parasitol. Res. 107: 261-269.

Siegfried E., Ochs H. \& Deplazes P. 2004. Clinical development and serological antibody responses in sheep and rabbits experimentally infested with Psoroptesovis and Psoroptescuniculi. Vet. Parasitol. 124:(1):109124.

Wagner R. \& Wendlberger U. 2000. Field efficacy of moxidectin in dogs and rabbits naturally infested with Sarcoptes spp., Demodex spp. and Psoroptes spp. mites. Vet. Parasitol. 93(2):149-158.

Wall R. \& Kolbe K. 2006. Taxonomic priority in psoroptic mange mites: $P$. ovis or P. equi? Exp. Appl. Acarol. 39(2):159-162.

Wall R. 2007. Ectoparasites: Future challenges in a changing World. Vet. Parasitol. 148(1):62-74.

White S.D., Bourdeau P.J. \& Meredith A. 2002. Dermatologic problems of rabbits. Sem. Avian and Exotic Pet Med. 11(3):141-150. 